\title{
Frecuencia e indicaciones de cesáreas electivas y de urgencia en el Hospital Regional de Ayacucho, Perú
}

\author{
Frequency and indications of elective and emergency cesarean sections in the \\ Hospital Regional de Ayacucho, Peru
}

Roaldo Pino-Anaya ${ }^{1, a}$, Vilma Zorrilla-Delgado ${ }^{\text {1,a, }}$, Maritza Rodríguez-Lizana ${ }^{\star 1, b}$, Walter Wilfredo Ochoa-Yupanqui ${ }^{1, c}$

Filiación y grado académico

Universidad Nacional de San Cristóbal de Huamanga, Ayacucho, Perú.

Maestro en Salud Pública.

Doctora en Educación.

Maestro en Docencia Universitaria.

D. ORCID iD de Roaldo Pino-Anaya https://orcid.org/0000-0002-8199-3731

ID. ORCID iD de Vilma Zorrilla-Delgado https://orcid.org/0000-0002-9930-1190

(D. ORCID iD de Maritza Rodríguez-Lizana https://orcid.org/0000-0003-2634-3418

(1) ORCID iD de Walter Ochoa-Yupanqui https://orcid.org/0000-0001-6591-4530

Contribución de los autores

RPA: conceptualización, administración del proyecto, investigación y validación.

VZD: conceptualización. Investigación y metodología.

MRL: validación, redacción del borrador original escritura (revisión y edición).

WwoY: software, análisis formal, escritura (revisión y edición).

Fuentes de financiamiento

La investigación fue financiada por la Oficina de Investigación e Innovación de la Universidad Nacional de San Cristóbal de Huamanga.

Conflictos de interés

Los autores declaran no tener conflicto de interés.

Recibido: 11-11-2020

Arbitrado por pares

Aceptado: 01-01-2021

Citar como

Pino-Anaya R, Zorrilla-Delgado V, Rodríguez-Lizana $M$, Ochoa-Yupanqui WW. Frecuencia e indicaciones de cesáreas electivas y de urgencia en el hospital docente. Ayacucho, Perú. Rev Peru Cienc Salud. 2021; 3(1): 32-7. doi: https://doi. org/10.37711/rpcs.2021.3.1.264

Correspondencia

Maritza Rodríguez Lizana

Dirección: Ayacuho, Perú

Telf.: 966100450

Email: Maritza.rodriguez@unsch.edu.pe

\section{RESUMEN}

Objetivo. Conocer la frecuencia e indicaciones de cesáreas electivas y de urgencia en relación a factores asociados en el Hospital Regional de Ayacucho. Métodos. Se realizó una investigación básica de diseño no experimental, descriptivo, transversal, prospectivo, correlacional de enfoque cuantitativo, con una muestra no probabilística e intencionada de 94 puérperas poscesareadas, previo consentimiento informado. Para la recolección de datos se utilizó la técnica de la encuesta y como instrumento el cuestionario y las historias clínicas para indagar el motivo de la cesárea. En el análisis inferencial se utilizó la prueba de chi-cuadrado al $95 \%$ de confianza $(p \leq 0,05)$. Resultados. El 51,8 \% (1455) de partos fueron por cesárea, siendo las indicaciones más frecuentes la cesárea anterior con el $17,2 \%$ de los casos, desproporción céfalo pélvica con el 13,7 \%, oligohidramnios con el $11,3 \%$ y otros en menores porcentajes; asimismo, el 59,6 \% de cesáreas son de urgencia y $40,4 \%$ electivas. No hay significancia $p>0,05$ entre las cesáreas y los factores paridad, edad materna y condición socioeconómica. Conclusiones. La frecuencia de cesáreas supera el $50 \%$, teniendo como primeras indicaciones la cesárea anterior, desproporción céfalo pélvica, oligohidramnios y sufrimiento fetal agudo. Los tipos de cesáreas que más se realizan son las de urgencia y las electivas. No existe significancia entre las cesáreas y paridad, edad materna y la condición socioeconómica.

Palabras clave: cesárea; histerotomía; frecuencia; indicaciones; hospital (Fuente: DeCS-BIREME).

\section{ABSTRACT}

Objective. To determine the frequency and indications of elective and emergency caesarean sections in relation to associated factors in the Hospital Regional de Ayacucho. Methods. A basic research of nonexperimental, descriptive, cross-sectional, prospective, correlational design with a quantitative approach was carried out, with a non-probabilistic and intentional sample of 94 post-cessation puerperal women, with prior informed consent. The survey technique was used for data collection, and the questionnaire and medical records were used as instruments to investigate the reason for cesarean section. In the inferential analysis, the chi-square test was used at 95\% confidence ( $p \leq 0.05)$. Results. 51.8\% (1455) of deliveries were by cesarean section, the most frequent indications being previous cesarean section with $17.2 \%$ of cases, cephalopelvic disproportion with $13.7 \%$, oligohydramnios with $11.3 \%$ and others in lower percentages; likewise, $59.6 \%$ of cesarean sections were emergency and $40.4 \%$ elective. There is no significance $p>0.05$ between cesarean sections and the factors parity, maternal age and socioeconomic status. Conclusions. The frequency of cesarean sections exceeds $50 \%$, with the first indications being previous cesarean section, cephalopelvic disproportion, oligohydramnios and acute fetal distress. The most common types of cesarean section are emergency and elective. There is no significance between cesarean sections and parity, maternal age and socioeconomic status.

Keywords: cesarean section; hysterotomy; frequency; indications; hospital (Source: MeSH - NLM). 


\section{INTRODUCCIÓN}

La cesárea es un procedimiento quirúrgico ${ }^{(1)}$ que conduce al parto mediante la incisión en las paredes abdominal (laparotomía) y uterina (histerotomía) ${ }^{(2-5)}$ y tiene por objeto disminuir la morbimortalidad materna perinatal, cuando el parto vaginal se complica ${ }^{(6)}$. Ya en 1985, la Organización Mundial de la Salud (OMS) estableció que la tasa ideal de cesárea debería oscilar entre el 10 \% y el $15 \%$, empleándose sólo para garantizar condiciones saludables al binomio madre-hijo, aunque hay tendencia al incremento en diversos países ${ }^{(7)}$, una tendencia que se observa también en la ciudad Ayacucho. Este incremento se debe a factores como: evolución de la cirugía y técnicas anestésicas, riesgos disminuidos de complicaciones posoperatorias inmediatas y las características de la organización del sistema de salud ${ }^{(1)}$, además de las exigencias de las mismas pacientes ${ }^{(2,3)}$.

Pese a que se percibe la cesárea como una forma de facilitar el trabajo de parto, siendo recomendada por los profesionales encargados y preferida por muchas gestantes, diversos investigadores advierten del riesgo que significa ${ }^{(8)}$, considerándose como un problema de salud pública ${ }^{(5)}$ porque aumenta el riesgo de morbimortalidad materna y perinatal ${ }^{(9)}$. Reportes recientes indican además que se realiza una cada 60 minutos $^{(8)}$.

La cesárea, justificada desde el punto de vista médico, es eficaz para prevenir la morbimortalidad materna y perinatal ${ }^{(7)}$. Las indicaciones más frecuentes se deben a la desproporción cefalopélvica, inducción fallida, presentación pelviana y sufrimiento fetal; sin embargo, su avance y desarrollo ha conducido a un uso irracional ${ }^{(10)}$, debido a: 1) la programación por los especialistas para asegurarse un parto corto y programarse en el trabajo con anterioridad; 2 ) el pedido directo de la gestante que desea un parto sin dolor; 3 ) la necesidad del entrenamiento de médicos residentes, pudiendo causar complicaciones en el acto operatorio y los efectos de la anestesia, ya que una cesárea aumenta seis veces el riesgo de muerte en relación al parto vaginal ${ }^{(11)}$. Más aun, para algunos colectivos estas transgreden asimismo los derechos reproductivos de las mujeres ${ }^{(12) .}$

Las complicaciones más frecuentes relacionadas a la cesárea varían según las distintas series y circunstancias, siendo las principales: la infección de herida operatoria $(0,7$ a $7,5 \%)$, transfusión sanguínea (5,3\%), hemorragia puerperal (3,1\%), endomiometritis (1,6\% a $8,3 \%)$, desgarro de histerorrafia y lesión urinaria e intestinal ${ }^{(13)}$; sin embargo, los efectos que tienen las tasas de cesárea sobre otros resultados pediátricos no están del todo claros y se necesitan investigaciones adicionales para comprender los efectos sobre algunos resultados inmediatos y futuros ${ }^{(14)}$. Entre las desventajas, se destaca el retraso del primer contacto madre-hijo, y cuando se produce, la madre está somnolienta y cansada, obstaculiza el inicio de la lactancia y la bajada de la leche tarda más en las madres que han parido por cesárea ${ }^{(15)}$.

En la decisión de emplear la cesárea debe primar la bioética que se relaciona con el uso excesivo y en ocasiones innecesario de la operación cesárea, que debe ser usada únicamente cuando el parto por vía vaginal sea riesgoso. En la actualidad, ya no es una decisión que toma de manera exclusiva y razonada el médico, sino que incluso es una opción que el mismo paciente elige ${ }^{(16)}$. Definitivamente se debe emplear solo cuando se sospeche que el parto vaginal sea un riesgo, tanto para la madre como para el niño ${ }^{(17)}$.

Según el criterio, las cesáreas pueden ser: a) absolutas, cuando las morbilidades o comorbilidades propias al embarazo-parto o cuyo diagnóstico indica que el parto vaginal no es posible, con altísimas probabilidades de muerte materna o fetal en caso de ocurrir un parto vaginal, y b) relativas, cuando se tiene en cuenta patologías que ameritan unas condiciones de atención (infraestructura, recurso humano, apoyos diagnósticos, etc.) que, de no tenerse, no permiten garantizar plenamente un buen pronóstico de la salud del binomio madre-feto durante la atención del parto vaginal ${ }^{(18)}$.

La tasa de cesáreas va en aumento, debido a que muchos profesionales consideran que se debe alcanzar el $15 \%$ que establece la $\mathrm{OMS}^{(19)}$ y, pese a los esfuerzos para reducir esta tasa, no hay resultados alentadores; muy al margen de ser practicada para disminuir la mortalidad materno neonatal ${ }^{(21)}$. Este incremento, que se traduce en que el $18,6 \%$ de todos los nacimientos se producen por cesáreas, y en regiones más desarrolladas llegan al 27,2 \% a nivel mundial, se denomina "epidemia de cesáreas" (20). Con base en los datos de 121 países, entre 1990 y 2014, la tasa promedio de cesárea global aumentó un 12,4\% (del 6,7\% al 19,1\%), siendo América Latina y el Caribe la región dónde ha habido un mayor incremento, con un $19,4 \%$ (del $22,8 \%$ al $42,2 \%)^{(22)}$.

La tasa de cesáreas en el Perú viene incrementándose desde 1992, el 20 \%, superando ampliamente el límite recomendado por la Organización Mundial de la Salud ${ }^{(20)}$. El Instituto Nacional de Estadística e Informática (INEI) reportó que la tasa de cesárea para el año 2017 fue de $40,8 \%{ }^{(23)}$. No hay evidencia de que la cesárea otorgue mayores beneficios y menores riesgos que el parto vaginal espontáneo a corto plazo; tampoco se encuentran estudios que evalúen la frecuencia de complicaciones a largo plazo, tales como placenta previa, acretismo placentario y embarazo ectópico en la cicatriz de la cesárea previa ${ }^{(24)}$, por lo que el objetivo de la presente investigación fue 
conocer la frecuencia e indicaciones de parto por cesárea en el Hospital Regional de Ayacucho. De este modo, se podrán plantear con posterioridad estrategias para su correcta utilización en beneficio del binomio madre-hijo.

\section{MÉTODOS}

\section{Tipo de estudio}

El tipo de investigación fue básico, de nivel descriptivo y diseño no experimental, transversal, prospectivo y correlacional. El estudio fue además de enfoque cuantitativo ${ }^{(25)}$ y se desarrolló en el Departamento de Gíneco-Obstetricia del Hospital Regional de Ayacucho (HRA).

\section{Población y muestra}

La población estuvo conformada por 1455 cesáreas del HRA; mediante un muestreo no probabilístico y la técnica intencionada, se seleccionó una muestra 94 puérperas poscesareadas, quienes, a su vez, firmaron un consentimiento informado.

\section{Instrumentos de recolección de datos}

La técnica empleada fue la encuesta y como instrumento se utilizó el cuestionario que tuvo 12 reactivos entre factores socioeconómicos (edad y condición socioeconómica) y antecedentes gineco-obstétricos (paridad). La validación se hizo mediante la $r$ de Pearson, logrando valores superiores a 0,21 , por reactivo, demostrando su validez.

\section{Procedimientos de la recolección de datos}

Luego de obtenida la autorización para la ejecución de la investigación por parte del director del HRA, se solicitó autorización a la jefatura del Departamento de GínecoObstetricia para la recolección de datos. Posteriormente, previa charla informativa, se aplicó el consentimiento informado a todas las puérperas poscesareadas. Luego, se recabó un cuestionario para obtener la información sobre factores sociodemográficos y gineco-obstétricos y las historias clínicas, a fin de determinar el motivo de la decisión de la cesárea; teniendo en cuenta: antecedentes obstétricos y control prenatal. Todo lo anterior tuvo lugar durante el periodo de abril a junio de 2019.

\section{Aspectos éticos}

La investigación respetó los principios éticos de: a). Autonomía: las gestantes decidieron participar de la

Tabla 1. Tipo de partos atendidos en el HRA, 2019

\begin{tabular}{lcc}
\hline Tipo de parto & $\mathbf{f i}$ & $\%$ \\
\hline Vaginal & 1351 & 48,2 \\
Cesárea & 1455 & 51,8 \\
Total & 2806 & 100,0 \\
\hline
\end{tabular}

Tabla 2. Indicaciones de cesárea en el HRA, 2019

\begin{tabular}{llc}
\hline Indicaciones & $\mathbf{f i}$ & $\%$ \\
\hline Cesárea anterior & 16 & 17,2 \\
Desproporción feto pélvica & 13 & 13,7 \\
Oligohidramnios & 11 & 11,3 \\
Sufrimiento fetal agudo & 9 & 9,8 \\
Embarazo gemelar & 8 & 8,7 \\
Presentación podálica & 8 & 8,2 \\
Pre eclampsia & 6 & 6,4 \\
Enfermedades maternas intercurrentes & 4 & 4,4 \\
Ruptura prematura de membranas & 3 & 2,9 \\
Trabajo de parto complicado por cordón & 2 & 2,5 \\
Situación transversa & 2 & 1,9 \\
Placenta previa & 2 & 1,6 \\
Parto prolongado & 2 & 1,6 \\
Otras patologías & 9 & 9,6 \\
Total & 94 & 100,0 \\
\hline
\end{tabular}

investigación mediante la firma del consentimiento informado, privilegiando la privacidad y la cultura; la aplicación del instrumento no causó daño alguno. b). Justicia: todas las gestantes recibieron el mismo trato y los resultados sirvieron solo para uso de la investigación. Se remarca que la investigación brinda información que se presenta solo para cumplir los objetivos del estudio, enmarcados en la Conducta Responsable de Investigación.

\section{Análisis de los datos}

Los datos obtenidos se codificaron y trasladados a una base de datos, según las variables establecidas, empleando el programa estadístico SPSS, V.24 y la hoja de cálculo Microsoft Excel, presentándose en tablas y gráficos según los objetivos a fin de facilitar su interpretación. Para el análisis inferencial, se utilizó la prueba chi cuadrado $\left(x^{2}\right)$ al $95 \%$ de confianza $(p<0,05)$.

\section{RESULTADOS}

La investigación evidencia que, de 2806 partos atendidos en el HRA, 1455 (51,8 \%) fueron sometidos a cesárea y un menor porcentaje $(48,2 \%)$ corresponde al parto vaginal tal como se visualiza en la tabla 1.

En cuanto a los motivos o indicaciones para proceder a la cesárea, se encontró que 16 casos $(17,2 \%)$ se

Tabla 3. Tipos de cesárea en el HRA, 2019

\begin{tabular}{lcc}
\hline Tipo de cesárea & $\mathbf{f i}$ & $\%$ \\
\hline Electiva & 38 & 40,4 \\
Urgencia & 56 & 59,6 \\
Total & 94 & 100,0 \\
\hline
\end{tabular}


Tabla 4. Tipos de cesárea en relación a los antecedentes gineco-obstétricos en el HRA, 2019

\begin{tabular}{|c|c|c|c|c|c|c|c|c|}
\hline \multirow{3}{*}{$\begin{array}{l}\text { Antecedentes } \\
\text { gineco-obstétricos }\end{array}$} & \multicolumn{4}{|c|}{ Cesárea } & \multirow{2}{*}{\multicolumn{2}{|c|}{ Total }} & \multirow{3}{*}{$x^{2}$} & \multirow{3}{*}{ p valor } \\
\hline & \multicolumn{2}{|c|}{ Electiva } & \multicolumn{2}{|c|}{ Urgencia } & & & & \\
\hline & fi & $\%$ & fi & $\%$ & fi & $\%$ & & \\
\hline Primípara & 9 & 9,6 & 13 & 13,8 & 22 & 23,4 & & \\
\hline Multípara & 29 & 30,9 & 43 & 45,7 & 72 & 76,6 & $\begin{array}{l}X^{2} c=0,003 \\
X^{2} t=3,841\end{array}$ & $p>0.05$ \\
\hline Total & 38 & 40,4 & 56 & 59,6 & 94 & 100,0 & & \\
\hline
\end{tabular}

debieron a una cesárea anterior, $13(13,7 \%)$ a la desproporción feto-pélvica, seguidos de 11 (11,3\%) a causa de oligohidramnios. También se reportan otras indicaciones como sufrimiento fetal agudo, embarazo gemelar, presentación podálica y otros en menores porcentajes (ver tabla 2).

La tabla 3 muestra la frecuencia de tipos de cesárea, enfatizando que 56 cesáreas $(59,6 \%)$ correspondieron a las de urgencia y $38(40,4 \%)$ a la cesárea electiva. En relación a los antecedentes gineco obstétricos de las mujeres que fueron sometidas a cesárea, 72 (76,6 \%) fueron multíparas y 22 (23,4\%) fueron primíparas (tabla 4). El estadístico chi cuadrado demuestra no significancia $\left(x^{2} c\right.$ =0,003); es decir, los tipos de cesárea no tienen relación con los antecedentes gineco-obstétricos, aceptando la hipótesis nula $(p>0,05)$ (ver tabla 4).

Respecto a los factores sociodemográficos: edad, 69 mujeres (73,4 \%) son adultas y $23(24,5 \%)$ añosas. La prueba chi cuadrado $\left(x^{2} c=5,178\right)$ revela que no hay significancia estadística. En cuanto a la condición socioeconómica, 85 mujeres $(90,4 \%)$ pertenecían al nivel medio y $9(9,6 \%)$ al nivel bajo. El estadístico chi cuadrado $\left(x^{2} c=0,208\right)$, indica que no hay significancia estadística (ver tabla 5), demostrando que los tipos de cesárea no se relacionan con la edad y la condición socioeconómica, confirmando la hipótesis nula $(p>0,05)$.

\section{DISCUSIÓN}

Las cesáreas superan lo recomendado por la OMS. En el HRA se practicaron un $51,8 \%$ de cesáreas durante el año 2019; siendo las indicaciones más frecuentes una cesárea anterior, desproporción feto-pélvica, oligohidramnios, entre otros. Sobre los tipos de cesáreas, predominan las de urgencia $59,6 \%$ y la cesárea electiva $40,4 \%$; en especial esta última debido, tal vez, a la información que tienen las gestantes o la inducción del personal de salud, sin tener en cuenta los riesgos y complicaciones. Por lo tanto, es necesario determinar con claridad los beneficios y el riesgo de esta práctica que permitan sustentar su uso ${ }^{(26)}$.

Diversos investigadores coinciden con nuestros hallazgos, reportando cesáreas superiores al $40 \%$ en América Latina y el Perú ${ }^{(18,22,27,28)}$, sustentando sus resultados en la preferencia por los especialistas y el pedido expreso de las propias gestantes ${ }^{(3,11)}$. En otras investigaciones, a diferencia de nuestro estudio, se encuentra un mayor uso de cesáreas en primigestas (21), aunque coinciden con las indicaciones de cesárea

Tabla 5. Tipos de cesárea en relación a los factores sociodemográficos en el HRA, 2019

\begin{tabular}{|c|c|c|c|c|c|c|c|c|}
\hline \multirow{3}{*}{$\begin{array}{l}\text { Factores } \\
\text { sociodemográficos }\end{array}$} & \multicolumn{4}{|c|}{ Cesárea } & \multirow{2}{*}{\multicolumn{2}{|c|}{ Total }} & \multirow{3}{*}{$\mathbf{x}^{2}$} & \multirow{3}{*}{ p-valor } \\
\hline & \multicolumn{2}{|c|}{ Electiva } & \multicolumn{2}{|c|}{ Urgencia } & & & & \\
\hline & fi & $\%$ & fi & $\%$ & fi & $\%$ & & \\
\hline \multicolumn{9}{|l|}{ Edad } \\
\hline Adolescente & 2 & 2,1 & 0 & 0 & 2 & 2,1 & \multirow{4}{*}{$\begin{array}{l}X^{2} c=5,178 \\
X^{2} t=5,991\end{array}$} & \multirow{4}{*}{$p>0.05$} \\
\hline Adulta & 24 & 25,5 & 45 & 47,9 & 69 & 73,4 & & \\
\hline Añosa & 12 & 12,8 & 11 & 11,7 & 23 & 24,5 & & \\
\hline Total & 38 & 40,4 & 56 & 59,6 & 94 & 100,0 & & \\
\hline \multicolumn{9}{|c|}{ Condición socioeconómica } \\
\hline Baja & 3 & 3,2 & 6 & 6,4 & 9 & 9,6 & \multirow{3}{*}{$\begin{array}{l}X^{2} c=0,208 \\
X^{2} t=3,841\end{array}$} & \multirow{3}{*}{$p>0.05$} \\
\hline Media & 35 & 37,2 & 50 & 53,2 & 85 & 90,4 & & \\
\hline Total & 38 & 40,4 & 56 & 59,6 & 94 & 100,0 & & \\
\hline
\end{tabular}


destacando la cesárea, destacando anterior ${ }^{(21)}$. Asimismo, aplicando el índice de Robson ${ }^{(17)}$ reportan que las indicaciones más frecuentes de operación cesárea fueron macrosomía fetal o desproporción feto-pélvica, oligohidramnios (15,1\%), sufrimiento fetal, el antecedente de más de dos cesáreas (1), cesárea previa, desproporción cefalopélvica ${ }^{(27)}$, sufrimiento fetal agudo ${ }^{(28)}$ y síndromes hipertensivos del embarazo (29); siendo necesario priorizar estos grupos para la cesárea (30), otros inciden en la paridad (pocos hijos) ${ }^{(5)}$.

No existe relación significativa entre la cesárea electiva y de urgencia con la paridad, edad y condición socioeconómica. A nivel mundial, se reporta una mayor frecuencia entre menores de 20 y mayores de 34 años de edad, primíparas, habitantes de áreas urbanas, con altos niveles de escolaridad y de estatus socioeconómico alto ${ }^{(12)}$, siendo similar a nuestra realidad. Investigaciones recientes han encontrado además una relación entre los indicadores socioeconómicos y el número de cesáreas, demostrando que las mujeres de nivel socioeconómico medio y alto tienen preferencia por el parto quirúrgico ${ }^{(9,26)}$, posiblemente porque tienen mejor acceso a los servicios prenatales ${ }^{(31)}$.

Finalmente, se concluye que en el HRA la frecuencia de cesáreas supera el $50 \%$ siendo las indicaciones principales la cesárea anterior, desproporción céfalopélvica, oligohidramnios y sufrimiento fetal agudo. El tipo de cesárea que más se realiza son las cesáreas de urgencia, electivas o programadas. No existe evidencia significativa $(p>0,05)$ de la relación entre las cesáreas y los factores asociados como la paridad, edad materna y la condición socioeconómica. Reafírmanos, por consiguiente, la necesidad de reducir la frecuencia de cesáreas mediante enfoques comunitarios e incidir en la capacitación para su uso únicamente en aquellos casos donde sea necesaria su realización.

\section{Agradecimientos}

A la obstetra Rosalía Gabino Candía que labora en el sistema informático perinatal (SIP) del Servicio de GinecoObstetricia y a las internas de Obstetricia del Hospital Regional de Ayacucho.

\section{REFERENCIAS}

1. Sanches NC; Mamede FV, VIVANCOS RBZ. Perfil Perfil das mulheres submetidas à cesareana e assistência obstétrica na maternidade pública em Ribeirão Preto. Texto contexto - enfrem [Internet].2012;21(2):418-426 [Consultado 2021 Ene 31] Disponible en https://doi.org/10.1590/ S0104-07072012000200021.

2. Anaya A, Londoño F, Pérez L, Ortiz R. Caracterización de las pacientes llevadas a cesárea según modelo de Robson y exploración de factores asociados en gestantes atendi- das en el hospital Universitario San José de la ciudad de Popayán-Colombia. 1 de enero de 2016 a 30 de junio de 2016. Rev chil Obs ginecol [Internet]. 2017; 82(5): 491-503 [Consultado 2021 Feb 4] Disponible en: https://scielo.conicyt.cl/scielo.php?pid=S0717-75262017000500491\&script=sci_arttext\&tlng=e

3. Hiralda J, Pérez A, Velázquez V. Comportamiento de la cesárea en pacientes del Hospital Vladimir Ilich Lenin de Holguín. Correo Científico Médico de Holguín [Internet] 2015; 19(4): 615-27 [Consultado 2021 Feb 4] Disponible en: http://scielo.sld.cu/pdf/ccm/v19n4/ccm03415.pdf

4. Martínez-Salazar G, Grimaldo-Valenzuela P, Vázquez-Peña G, Reyes-Segovia C, Torres-Luna G, Escudero-Lourdes G. Operación cesárea. Una visión histórica, epidemiológica y ética para disminuir su incidencia. Rev Med Inst Mex Seguro Soc [Internet].2015; 53(5): 608-615 [Consultado 2021 Feb 4];:Disponible en: https://www.redalyc. org/pdf/4577/457744939018.pdf

5. Sinchitullo-Castillo A, Roldán-Arbieto L, Arango-Ochante $P$. Factores asociados a partos por cesáreas en un Hospital Peruano. Rev Fac Med Hum [Internet].2020; 20(3):444-51 [Consultado 2021 Feb 6] Disponible en: http://www.scielo.org.pe/pdf/rfmh/v20n3/2308-0531rfmh-20-03-444.pdf

6. Ortiz RA, Moreno E, Mambuscay J, Muñoz J. Prevalencia de complicaciones en pacientes sometidas a cesárea en el periodo comprendido entre enero a diciembre del 2016 en el Hospital Universitario San José de Popayán, Colombia 2016. Rev chil Obs ginecolol [Internet].2019; 84(6):435-48 [Consultado 2021 Feb 4].Disponible en: https://scielo.conicyt.cl/scielo.php?script=sci_arttext\&pi$\mathrm{d}=$ S0717-75262019000600435

7. OMS. Declaración de la OMS sobre tasas de cesáreas [Internet]. Ginebra; 2015 [Consultado 2021 Ene 12] Disponible en: https://apps.who.int/iris/bitstream/ handle/10665/161444/WHO_RHR_15.02_spa.pdf;jsessionid=E0405BAD0C428FABF721B17A48ED7A1B?sequence $=1$

8. Hernández J, Martínez F, Mojena Y. Caracterización de cesáreas primitivas en Santa Cruz del Norte. Rev Cubana Obstet Ginecol [Internet]. 2017; 43(3):6679 [Consultado 2021 Feb 4] Disponible en: http:// scielo.sld.cu/scielo.php?script=sci_arttext\&pi$d=$ S0138-600X2017000300007

9. Zuleta-Tobon J, Quintero-Rincón F, Quiceno-Ceballos A. Aplicación del modelo de Robson para caracterizar la realización de cesáreas en una institución de tercer nivel de atención en Medellín, Colombia: Estudio de corte transversal. Rev Colomb Obstet Gineco [Internet]. 2013; 64(2): 90-99 [Consultado 2021 Ene 21] Disponible en: http://www.scielo.org.co/scielo.php?script=sci_abstract\&pid=S0034-74342013000200002\&lng=en\&nrm=iso\&tlng=es

10. Nápoles CD, Piloto-Padrón M. Consideraciones actuales sobre la operación cesárea. MEDISAN [Internet]. 2012; 16(10):1595 [Consultado 2021 Feb 4] Disponible en: http://scielo.sld.cu/scielo.php?script=sci_arttext\&pi$\mathrm{d}=\mathrm{S} 1029-30192012001000013$

11. Bobadilla LE, León F. En torno a las cesáreas en el Perú: ¿solo un problema de cifras? Rev Peru Ginecol [Internet]. 2017; 63(4):659-60 [Consultado 2021 Feb 4] Disponible en: http://www.spog.org.pe/web/revista/index.php/ RPGO/article/view/2044 
12. Suárez-López L, Campero L, De la Vara-Salazar E, Rivera-Rivera L, Hernández-Serrato $M$, Walker D, et al. Características sociodemográficas y reproductivas asociadas con el aumento de cesáreas en México. Salud Publica Mex [Internet]. 2013; 55(2):S225-34 [Consultado 2021 Feb 4];. Disponible en: http://www.scielo.org.mx/pdf/ spm/v55s2/v55s2a20.pdf

13. Puma J, Díaz J, Caparó C. Complicaciones maternas de la cesárea en gestantes a término en periodo expulsivo en un hospital general de Lima, Perú. Rev Med Hered [Internet].2015; 26(1):17-23 [Consultado 2021 Feb 4] Disponible en: http://www.scielo.org.pe/scielo.php?pi$\mathrm{d}=$ S1018-130X2015000100004\&script=sci_arttext\&tln$\mathrm{g}=\mathrm{en}$

14. Sadler M. Los efectos de la cesárea en la salud infantil: Un asunto urgente. Rev chil pediatr [Internet]. 2018; 89(4):561-2 [Consultado 2021 Feb 4] Disponible en: https://scielo.conicyt.cl/scielo.php?pi$\mathrm{d}=$ S0370-41062018000400561\&script=sci_arttext\&tIn$\mathrm{g}=\mathrm{en}$

15. Uzcátegui O, Cabrera C. Aspectos bioéticos de la cesárea y consentimiento informado. Rev Obs Ginecol Venez [Internet].2010; 70(1):47-52 [Consultado 2021 Feb 4] Disponible en: http://ve.scielo.org/scielo.php?script=sci arttext\&pid=S0048-77322010000100009

16. Vázquez J. Abuso de la operación cesárea y el principio de beneficencia. rev.latinoam.bioet [Internet].2015; 16(1): 60-71 [Consultado 2021 Feb 4] doi: http://dx.doi. org/10.18359/rlbi.1441

17. Morgan-Ortiz F, Luna-Hernández K, Peraza-Garay $F$, Morgan-Ruiz F, Martínez-Román J, Corona-Sapien C, et al. Frecuencia de las indicaciones de operación cesárea de acuerdo con la clasificación de robson. Rev Med UAS [Internet]. 2018; 8(1):20-30 [Consultado 2021 Ene 20];. Disponible en: http://dx.doi.org/10.28960/revmeduas.2007-8013.v8.n1.003

18. Luque AS. Indicaciones y tasa de cesáreas en el Hospital EsSalud III Puno en el año 2016 [Internet]. Puno: Universidad Nacional del Altiplano; 2017 [Consultado 2021 Ene 14]. Disponible en: http://repositorio.unap.edu.pe/ bitstream/handle/UNAP/3851/Luque_Coyla_Alexander_Salvio.pdf?sequence $=1$ \&isAllowed $=y$

19. Vallejos A, Espino S, Jaimes L, Zepeda C, Cabrera DA, Arriaga $L$, et al. Actitudes de los obstetras sobre el parto por cesárea: un estudio en hospitales de la ciudad de México. Perinatol Reprod Hum [Internet]. 2018; 32(1):19-26 [Consultado 2021 Feb 4] Disponible en: https://www.sciencedirect.com/science/article/pii/ S0187533718300359

20. Quispe A, Santivañez-Pimentel Á, Leyton-Valencia I, Pomasunco D. Cesáreas en siete hospitales públicos de Lima: análisis de tendencia en el periodo 2001-2008. Rev perú med exp salud publica [Internet] 2010; 27(1): 45-50 [Consultado 2021 Ene 21] Disponible en: http:// www.scielo.org.pe/scielo.php?script=sci_arttext\&pi$\mathrm{d}=\mathrm{S} 1726-46342010000100008$

21. Rosado-Gorocica JD, Montoya-López S, Dzul-Rosado KR, Zavala-Castro JE, Puerto FI. Frecuencia, indicaciones y conocimiento de embarazadas sobre la cesárea: El caso de un hospital de la ciudad de Mérida, Yucatán. Rev bio- med [Internet] 2013; 24(3):100-108 [Consultado 2021 Ene 31] Disponible en: https://www.revistabiomedica. $\mathrm{mx} /$ index.php/revbiomed/article/view/62

22. Betrán AP, Ye J, Moller A-B, Zhang J, Gülmezoglu AM, Torloni MR. La tendencia creciente en las tasas de cesárea: estimaciones mundiales, regionales y nacionales: 1990-2014. Zeeb H, editor. PLoS One [Internet] 2016; 11(2):e0148343 [Consultado 2021 Ene 27] Disponible en: https://doi.org/10.1371/journal.pone.0148343

23. Perú. Instituto Nacional de Estadística e Informática. Encuesta demográfica y de salud familiar - ENDES 2017. Nacional y Departamental [Internet] [Consultado 2021 Feb 2] Disponible en: https://www.inei.gob.pe/media/ MenuRecursivo/publicaciones_digitales/Est/Lib1525/ index.html

24. Fonseca-Pérez JE. Cesárea por solicitud materna (CPSM). Rev Colomb Obstet Ginecol [Internet].2017;68(1):7-11. [Consultado 2021 Feb 2] Disponible en: https://www. redalyc.org/pdf/1952/195250686001.pdf

25. Hernández R, Fernández C, Baptista P. Metodología de la Investigación [Internet]. 6a ed. México D.F: Editorial Mexicana; 2014 [Consultado 2021 Feb 7]. Disponible en: https://www.uca.ac.cr/wp-content/uploads/2017/10/ Investigacion.pdf

26. Ruiz-Sánchez J, Espino-Sosa S, Vallejos-Parés A, Durán-Arenas L. Cesárea: Tendencias y resultados. Perinatol Reprod Hum [Internet]. 2014; 28(1): 33-40 [Consultado 2021 Feb 4] Disponible en: http://www. scielo.org.mx/scielo.php?script=sci_arttext\&pi$\mathrm{d}=$ S0187-53372014000100006

27. Barrena M, Quispe P, Flores M, León C. Frecuencia e indicaciones del parto por cesárea en un hospital docente de Lima, Perú. Rev Peru Ginecol y Obstet [Internet] 2020; 66(2):1-6 [Consultado 2021 Ene 21] Disponible en: http://www.scielo.org.pe/scielo.php?script=sci_arttext\&pid=S2304-51322020000200004

28. Estrada-Aguilar C, Hernández-Carranza R, Gómez-Alonso $C$, Negrete-Paz M de L. Incidencia de cesareas. Rev Med Inst Mex Seguro Soc [Internet]. 2012; 50(5):517-22 [Consultado 2021 Feb 6] Disponible en: https://www.redalyc.org/pdf/4577/457745497012.pdf

29. Acevedo-Muñoz F, Jara-García J, Velásquez-Contreras I. Cesarea de urgencia: Características Biosociodemográficas de las madres intervenidas en esta modalidad de parto, Hospital Regional Valdivia [Internet].Valdivia: Universidad Austral de Chile; 2013 [Consultado 2021 Ene 14]. Disponible en: http://cybertesis.uach.cl/tesis/ uach/2013/fma173c/doc/fma173c.pdf

30. Jiménez-Hernández D, Guevara-Rodríguez A, Zuleta-Tobón J, Rubio-Romero J. Tasa de cesáreas por grupos de Robson en una Institución de Mediana Complejidad de la Ciudad de Bogotá, 2012-2014d. Rev Colomb Obstet Ginecol [Internet]. 2016; 67(2):101-11 [Consultado 2021 Feb 4] Disponible en: http://www.scielo.org.co/pdf/ rcog/v67n2/v67n2a03.pdf

31. Leona T, Padmadas S, Matthews Z. Factores comunitarios que afectan el aumento de las tasas de cesáreas en los países en desarrollo: un análisis de seis países. Soc Sci Med [Internet]. 2008; 67(8): 1236-46 [Consultado 2021 Ene 21] Disponible en: https://www.sciencedirect.com/science/ article/abs/pii/S0277953608003304?via\%3Dihub. 